\title{
La Intensificación Negociada: Cambios en el Régimen de Trabajo de una Gran Empresa de Retail en Chile (2006-2018)
}

\author{
Nicolas Ratto \\ ${ }^{1}$ Investigador independiente. Máster en Sociología por la Universidad de Chile. Santiago, Chile. \\ E-mail: nicolas.ratto@ug.uchile.cl.
}

\section{INTRODUCCIÓN}

La mayoría de los estudiosos del trabajo contemporáneos concordarían en caracterizar los últimos cuarenta años como tiempos de expansión progresiva de las formas de trabajo precario, de desregulación de las relaciones laborales, de privatización de la protección social y de declinación de la actividad sindical (De la Garza, 2000; Ikeler, 2019; Kelly, 2012; Visser, 2019). Si bien durante los años noventa el panorama en el campo estuvo plagado de fatalismos, de descripciones de las nuevas formas de organización del trabajo y de narrativas que sugerían la debilidad de los sindicatos y de los trabajadores ante los tiempos de cambios y globalización, paulatinamente desde el cambio de siglo las investigaciones se han abocado a estudiar los contramovimientos del trabajo ante la mercantilización de la sociedad (Ibsen y Tapia, 2017; Silver, 2003; Webster y O’Brien, 2020).

Esta contratendencia no se da solo como movimiento intelectual, sino también por el hallazgo de experiencias de revitalización sindical (Frege y Kelly, 2004) que han sido teorizadas en el marco de las estrategias de revitalización (Brookes, 2013; Dörre et al., 2009a; Ibsen y Tapia, 2017; Tattersall, 2013) o de movilización de recursos de poder (Fichter et al., 2018; Lévesque y Murray, 2010). Se suma que la mirada académica se ha ido descentrando de Europa y América del Norte, observando nuevas formas de organización en la periferia del movimiento de trabajadores, especialmente en el Sur Global (Webster y O'Brien, 2020); a la vez que se ha cuestionado la centralidad de la clase obrera industrial (De la Garza Toledo, 2009) y de la forma sin- 
dical como prototipo de forma de organización de los trabajadores y trabajadoras (Atzeni y Ness, 2018). Este cúmulo de investigaciones demuestra que, ante la precariedad laboral, los trabajadores suelen resistirse y organizarse, incluso cuando las labores que realizan y las condiciones de empleo en las que se encuentran aparentemente crean las condiciones perfectas para socavar la movilización colectiva.

Los efectos específicos que las acciones sindicales eventualmente generan sobre las condiciones de empleo y sobre los regímenes de producción que regulan el trabajo y sus conflictos han permanecido ocultos (Ikeler, 2019). Las investigaciones suelen presentar las experiencias sindicales que estudian como gestas heroicas e innovadoras, ocultando el cotidiano y lento trabajo político de muchos trabajadores en sus lugares de trabajo (Kenny, 2018). A su vez, las miradas profundas y sistemáticas que describen las experiencias de organización sindical no logran, o no deciden, cruzar el perímetro del centro de producción y observar el proceso a partir del cual el trabajo se organiza, se revela y vuelve a ser dominado. Así pues, los estudios de las estrategias sindicales y de las resistencias del trabajo (Fichter et al., 2018; Frege y Kelly, 2004; Kelly, 2012) han ido por un carril distinto y paralelo al de los procesos y regímenes de trabajo (Burawoy, 1985; Thompson, 2010). Según Ikeler (2019:2), "los estudiosos de la revitalización alejaron este debate del trabajo mismo. Si bien muchos se enfrentan al desafío de organizar a los trabajadores precarios, la precariedad y otros aspectos del "mal trabajo" a menudo se toman como condiciones estáticas $(. . .)^{\prime \prime}$. Por el lado de las teorías del proceso de trabajo se ha señalado la necesidad de considerar el interés y las "quejas" de los trabajadores en los análisis (Marks y Thompson, 2010), "pero han fallado en hacer referencias explícitas a la agencia colectiva y a los sindicatos, los que si bien se han debilitado deben seguir considerándose como mecanismos importantes que permiten la movilización" (Taylor y Moore, 2015:3).

La presente investigación pretende aportar en la reconciliación de las tradiciones teóricas de la revitalización sindical y de los procesos de trabajo, mediante el estudio en profundidad de la transición entre dos regímenes de trabajo en una gran empresa de retail en Chile entre los años 2006 y 2018. Ésta fue promovida por el efecto combinado de la creciente actividad sindical al interior de la empresa y la mayor regulación legal del trabajo en el sector, en el marco del crecimiento económico sostenido de la empresa, que la llevó a ocupar una posición monopólica entre los retailers nacionales. Este cambio en el régimen 
de trabajo se puede interpretar como un "intercambio" o "compromiso positivo de clase" (Wright, 2000) entre la empresa y las nuevas organizaciones sindicales que emergen en su interior en los primeros años de la década de 2000. El régimen de trabajo en la empresa pasó de uno "despótico" basado en la desprotección legal, la inseguridad salarial, el abuso y las prácticas antisindicales, a otro "hegemónico segmentado" con mayores protecciones legales, seguridad salarial diferenciada, buen trato, intentos de control normativo y fomento de la sindicalización. A cambio de estas concesiones, la empresa ha obtenido un relativo "consenso" por parte de los trabajadores y trabajadoras (desigual según los momentos y la organización sindical) con la intensificación sostenida de sus trabajos y la "sobreexplotación" de un segmento de los empleados de la empresa: aquellos con menor antigüedad y aquellos con los formatos de trabajo más precarios. Así, los sindicatos de la empresa parecen haber evitado y alcanzado, respectivamente, los "futuros" (Visser, 2019) de la marginalización y la revitalización sindical, siendo sus desafíos actuales los de la superación de la dualización de los mercados de trabajo en la empresa.

\section{MARCO DE REFERENCIA: LOS REGÍMENES DE TRABAJO}

El concepto de "régimen de producción" o de "régimen de trabajo" ha sido utilizado y desarrollado primera y progresivamente por Michael Burawoy $(1978,1983,1985,2014)$, en el marco de sus investigaciones de "caso extendido" en diversos centros de trabajo y de las discusiones que ha sostenido con la Labor Process Theory (LPT), la teoría marxista del proceso de trabajo. Estas discusiones teóricas han sido especialmente intensas con la "primera ola" de la LPT, cuyo máximo exponente fue Harry Braverman (1974) ${ }^{1}$. Si bien tanto Burawoy (1978) como otros críticos de Braverman al interior de la misma LPT (Friedman, 1977) valoraban el giro inaugurado por éste hacia el análisis de los procesos de producción y las dinámicas de control para la caracterización de las etapas del desarrollo del capitalismo, dudaban de que las tendencias capitalistas contemporáneas se movieran meramente hacia la descalificación y el creciente control sobre el trabajo. Además, le criticaban a Braverman el análisis de los procesos de trabajo y de control capitalista sin ninguna referencia a la subjetividad obrera: sus consentimientos, sus resistencias, sus miedos y sus arreglos.

En tal contexto de discusión teórica, y apoyado en sus investigaciones de realidades laborales tan diversas como las capitalistas de los siglos XIX y XX en Inglaterra y Estados Unidos, la soviética y la de países 
poscoloniales (Burawoy, 1983, 1985), el autor propone el concepto de "régimen de producción". Con este concepto "politiza" el estudio de las dinámicas de trabajo, por un lado, al entenderlas como procesos basados en antagonismos estructurados $y$, por ende, como realidades contestables que, de alguna manera, se reproducen "normalmente", conciliando los intereses antagónicos de las "clases sociales fundamentales": obreros y capital $^{2}$. Por otro lado, la politización de la producción teorizada por Burawoy va en la línea de trasladar un concepto propio del mundo de la política ("régimen") al mundo de la producción y las relaciones de clase. Los centros de producción, entonces, podrían pensarse como lugares de trabajo con sus propios "Estados" y "Mercados" al interior de un gran Estado a escala nacional, el cual, si bien afecta y regula estos lugares de trabajo, no implica la anulación de su autonomía.

Los dos regímenes clásicos identificados por Burawoy en el mundo del capital fueron el "despótico" y el "hegemónico". El despótico puede encontrar su prototipo en los regímenes de trabajo ingleses del siglo XIX descritos por Karl Marx en El Capital, en los cuales la extracción de esfuerzos se da principalmente a través de la coerción y el miedo al despido, en el contexto de un Estado débil, especialmente en términos de la regulación al trabajo y la seguridad social (Anner, 2015) y, de una anárquica competencia de los capitales, que los llevaría a intensificar continuamente el trabajo e introducir tecnologías en los procesos de producción (Burawoy, 1983). En este régimen los trabajadores tendrían un bajo poder de negociación debido al alto desempleo y las bajas regulaciones laborales, lo que los llevaría a aceptar malas condiciones de trabajo y a ser cautos en su organización sindical, por miedo a ser fácilmente despedidos.

Por el contrario, el régimen hegemónico se caracteriza por el desarrollo de un mercado de trabajo interno que privilegia la antigüedad de los empleados, lo que promueve en los obreros que sus intereses se desplacen a la longevidad y estabilidad en la empresa (Burawoy, 2014). Además, una "maquinaria de reclamos constituye obreros con derechos y obligaciones y cuyo aparato de negociación colectiva o paritarias coordina los intereses de los trabajadores y los gerentes" (Burawoy, 2014:161). En estas negociaciones colectivas los trabajadores serían representados por sindicatos que los protegen de despidos arbitrarios y reducciones salariales. En este régimen el Estado se ha tornado más fuerte, por lo que la seguridad social garantiza la reproducción 
de las fuerzas de trabajo a cierto nivel mínimo, independiente de su participación en la producción, como también el mismo Estado limita los métodos de la dominación gerencial (Burawoy, 1983).

El régimen de trabajo entonces sería un modo de regular el trabajo y el conflicto en la producción mediante aparatos políticos, ideológicos y económicos (Burawoy, 1985). Con regímenes de producción particulares, en base a arreglos de coerción y consenso, el capitalista conseguiría la materialización de la fuerza de trabajo en mercancías, que una vez vendidas le permitirían acumular capital y repetir así el proceso una y otra vez. ¿Por qué en algunos lugares y momentos se dan regímenes más despóticos y violentos, y en otros regímenes más consentidos y hegemónicos? Las respuestas desde la teoría apuntan a tres grandes factores: las regulaciones político-estatales (Burawoy, 1985; Nichols et al., 2004; Thompson y Broek, 2010), las dinámicas de competencia entre capitalistas (Burawoy, 1983, 1985; Nichols et al., 2004) y la organización colectiva de los trabajadores (Campbell, 2018; Nichols et al., 2004). Si bien los dos primeros factores "externos" a los regímenes de trabajo (Estado y mercado) están bastante presentes en los análisis de Burawoy (1985) y, de hecho, son parte fundamental para la definición de los particulares regímenes de trabajo, el tercer aspecto, el de la organización colectiva de los trabajadores, ha sido escasamente tematizado por el autor y por las investigaciones en general. Cuando el sujeto trabajador aparece en los esquemas de Burawoy (1985), lo hace más como factor de control del trabajo y de estabilización que como factor de cambio o ruptura.

Conocer los regímenes de producción en el comercio minorista o retail y el influjo que genera sobre estos la acción sindical tendría especial relevancia en tanto el sector es uno de los prototipos de las nuevas formas de trabajo precario en el neoliberalismo (Chan et al., 2019) y de mayor expansión en las últimas décadas al amparo de la liberalización económica (Harvey, 2007). Además, pese a la progresiva formación de clase en el sector (Bank, 2017; Ikeler, 2019; Kenny, 2018; Silver, 2003), hasta el momento no se ha reportado que las expresiones "desde abajo" del movimiento de trabajadores afecte a los regímenes de trabajo en las empresas, modifique las regulaciones estatales o revierta los procesos precarización y descalificación. Las resistencias de los trabajadores parecen no haber frenado la flexibilización precaria de los contratos y tiempos de trabajo, a través del uso de jornadas parciales, los contratos de corta duración y la tercerización del empleo (Chan et al., 2019; Ikeler, 
2019); ni tampoco la descalificación progresiva del trabajo, a través de la rutinización y de la erosión de su contenido conceptual (Curley y Royle, 2013; Ikeler, 2016; Stecher y Martinic, 2018).

\section{MARCO METODOLÓGICO}

Se llevó a cabo un estudio de caso (Coller, 2000) de las relaciones laborales al interior de una empresa, mediante la realización y análisis de entrevistas a trabajadores y dirigentes sindicales, la revisión documental y la construcción y análisis de bases de datos. El caso corresponde a una gran empresa del sector del comercio minorista (retail) supermercadista de Chile, la cual fue seleccionada por su importancia económica en el rubro (ver Cuadro 1), por la gran cantidad de fuerza de trabajo que emplea (más de 50.000 trabajadores y trabajadoras), por el carácter transnacional de su controlador (que hace el caso comparable internacionalmente) y por su alta tasa de sindicalización si comparada con los niveles del sector del comercio (casi un $70 \%$ contra un $20 \%$ ).

Las fuentes de información utilizadas fueron las siguientes:

1. Documentos: fuentes secundarias producidas por las organizaciones sindicales de la empresa, la empresa, el poder legislativo, los medios de comunicación e investigadores sociales. En particular, estas fuentes son contratos y convenios colectivos, historias de las Leyes, Leyes, Memorias y / o reportes anuales de la empresa, tesis y artículos científicos, notas de prensa, información de páginas webs o de Facebook, entre otras. Estos documentos se analizaron principalmente con las técnicas del análisis de contenido cualitativo, mediante el programa Atlas Ti.

2. Bases de datos: se analizaron bases de datos producidas por organismos públicos (CASEN, ENE, entre otras) y se analizaron otras de producción propia, como una base de datos de los 386 locales de la empresa y de la presencia sindical en cada uno de estos, otra de las características de su fuerza de trabajo y otra de las publicaciones en Facebook de las dos organizaciones sindicales más grandes de la empresa. Las primeras bases se analizaron mediante estadísticos descriptivos univariados y bivariados (con el programa SPSS) y 
las segundas se analizaron mediante herramientas cartográficas (con el programa Google Maps), y con las técnicas univariadas y bivariadas ya mencionadas.

3. Entrevistas: se realizaron entrevistas semi estructuradas o en profundidad a los principales dirigentes sindicales $(\mathrm{N}=3)$ de tres de las cuatro organizaciones sindicales más grandes de la empresa y a delegados sindicales de locales de la empresa $(\mathrm{N}=2)$. Estas entrevistas fueron transcritas y para el análisis se utilizó la técnica del análisis de contenido cualitativo mediante el software Atlas Ti. También se ocuparon entrevistas y extractos de entrevistas producidas en el marco de otras tesis o proyectos de investigación (Abarca, 2016; Contreras, 2015; Rayo, 2007).

\section{RESULTADOS}

\section{La empresa Walmart en Chile. Algunas características estructurales}

Antes de que Walmart llegara a Chile en 2009, sus locales eran administrados por un holding nacional. Las primeras formas supermercadistas de este holding se remontan al año 1957, con la apertura del primer supermercado autoservicio de Chile y América Latina. Desde mediados de los ochenta, en el contexto de un régimen de acumulación neoliberal ya consolidado en el país, la supermercadista nacional se irá expandiendo constantemente hasta llegar a ser comprada en 2008-2009 por la transnacional estadounidense. Actualmente la empresa tiene más de 380 locales a lo largo del país, en los cuales trabajan más de 50.000 empleados distribuidos en los cinco "formatos" o "marcas" de la empresa, que para simplificar serán numeradas como: FORMATO 1, FORMATO 2, FORMATO 3, FORMATO 4 y FORMATO 5. Chile se convierte, entonces, en uno de los más de veintiocho países en los que la empresa norteamericana tiene presencia, alimentando su masa de más de dos millones de trabajadores a nivel mundial (Bank et al., 2018).

Al menos desde el año 1998 hasta la actualidad, Walmart ha tenido un crecimiento sostenido en Chile: la cantidad de locales de la empresa creció en un 774\%, es decir, pasó de 50 a 387, la empresa pasó de dominar el 22,4\% del mercado de las grandes empresas supermercadistas 
de Chile en 1998 a dominar el 40\% en 2014 (posición casi monopólica ${ }^{3}$ ) y sus trabajadores aumentaron de 17.031 en 2001 a más de 50.000 en 2016 (Cuadro 1).

\section{Cuadro 1}

Número de locales, de empleados y porcentaje de participación en el mercado de la empresa (1998-2018)

\begin{tabular}{lccccccccccc}
\hline & 1998 & 2000 & 2002 & 2005 & 2007 & 2008 & 2010 & 2012 & 2014 & 2016 & 2018 \\
\hline Locales & 50 & 52 & 62 & 85 & 165 & 197 & 277 & 327 & 380 & 363 & 387 \\
\hline $\begin{array}{l}\text { Número de } \\
\text { empleados }\end{array}$ & - & $\begin{array}{l}17.031 \\
(2001)\end{array}$ & 21.800 & 46.775 & $\begin{array}{c}35.345 \\
(2006)\end{array}$ & - & - & - & - & $50.762 \begin{array}{r}50.716 \\
(2017)\end{array}$ \\
\hline $\begin{array}{l}\text { Participación de } \\
\text { ventas en mercado }\end{array}$ & $22,4 \%$ & $28,3 \%$ & - & $35,3 \%$ & - & $34 \%$ & - & - & $35 \%$ & $40 \%$ & - \\
\hline
\end{tabular}

Fuente: elaboración propia en base a Bank (2017:53), Feller-Rate (2009:6), Fundación Sol (2007), Reportes Anuales de la Empresa, La Tercera (2010) y producción de información propia.

Al año 2018 los trabajadores de la empresa se encuentran dispersos a lo largo del territorio nacional, en 387 locales y en los cinco formatos mencionados (Cuadro 2). Cada uno de estos formatos de negocios tiene diferencias sustantivas que hacen que en algunos de ellos sea especialmente difícil construir sindicalismo, por razones como el tamaño de cada local, la rotación laboral y la capacidad de obtener beneficios (dados los volúmenes de las ventas).

\section{Cuadro 2}

Los distintos formatos de Walmart en Chile. Año 2017

\begin{tabular}{lccccc}
\hline & $\begin{array}{c}\text { Formato 1: } \\
\text { Central } \\
\text { Mayorista }^{4}\end{array}$ & $\begin{array}{c}\text { Formato 2: } \\
\text { Hiper Líder }\end{array}$ & $\begin{array}{c}\text { Formato 3: } \\
\text { Líder Express }\end{array}$ & $\begin{array}{c}\text { Formato 4: } \\
\text { A Cuenta }^{5}\end{array}$ & $\begin{array}{c}\text { Formato 5: } \\
\text { Ekono }^{6}\end{array}$ \\
\hline Locales & 5 & 84 & 88 & 112 & 88 \\
\hline Productos & Alimentos & $\begin{array}{c}\text { Alimentos, hogar, } \\
\text { electrodomésticos, } \\
\text { electrónica, tex- } \\
\text { tiles y juguetes }\end{array}$ & Alimentos & Alimentos & Alimentos \\
\hline Proveedores & Marcas & Diversificados & Diversifica- & Marcas & Marcas \\
propias & dos & propias & propias \\
\hline Superficie & Alta & Alta & Media & Media & Baja \\
\hline Fuente: Walmart Chile (2017). & & & &
\end{tabular}




\section{LA IRRUPCIÓN SINDICAL EN LA EMPRESA}

Desde mediados de los años dos mil no solamente se transnacionaliza el capital controlador de la empresa (2009) y aumenta en más del doble su participación en el mercado, también emergen nuevos y cada vez más poderosos interlocutores representantes de los trabajadores (sindicatos), con los cuales la empresa tendrá que negociar e interactuar. Dos son las organizaciones más grandes en la empresa, La Federación del Trabajador de Walmart (LA FEDERACIÓN) y El Sindicato Interempresa de Líder de Walmart (EL SINDICATO). Estas organizaciones sindicales tienen su origen formal en 2007 y 2010 respectivamente, y agrupan actualmente cerca de 15.000 trabajadores cada una. En sus inicios estas organizaciones desarrollaron estrategias sindicales radicalmente diferentes ("paralelismo estratégico"): la primera movilizó principalmente su poder disruptivo mediante huelgas en el marco de negociaciones colectivas descentralizadas por local (2006-2012), y luego centralizadas, a nivel nacional (2014), mientras que la segunda organización optó por posturas más cercanas al diálogo social al nivel de empresa y al nivel de la política nacional (Ratto, 2019; ver Figura 1). En los últimos años las dos organizaciones sindicales han comenzado a poner en práctica, al nivel de la empresa, estrategias similares ("paralelismo ideológico"), con resultados parecidos, en el marco de la competencia por los socios y en la búsqueda de cada una por desprestigiar a la otra organización. En específico, se han centrado en la realización de negociaciones colectivas bianuales, en las que si bien amenazan con el uso de la huelga, ésta nunca se vuelve efectiva. Bastante más abajo que el SINDICATO y la FEDERACION en cuanto al número de socios, se ubican otras dos federaciones más pequeñas, con alrededor de 2.000 trabajadores y trabajadoras cada una. Además, existen algunos sindicatos "autónomos" de locales específicos, cuyas dimensiones están en torno a los 200 socios. En la empresa son más de 100 los sindicatos existentes (la legislación chilena permite que existan sindicatos por locales de trabajo), pero estos se tienden a agrupar en las tres federaciones ya comentadas. 
Figura 1

Línea de tiempo de la evolución del conflicto y sindicalismo en la empresa (2006-2018)

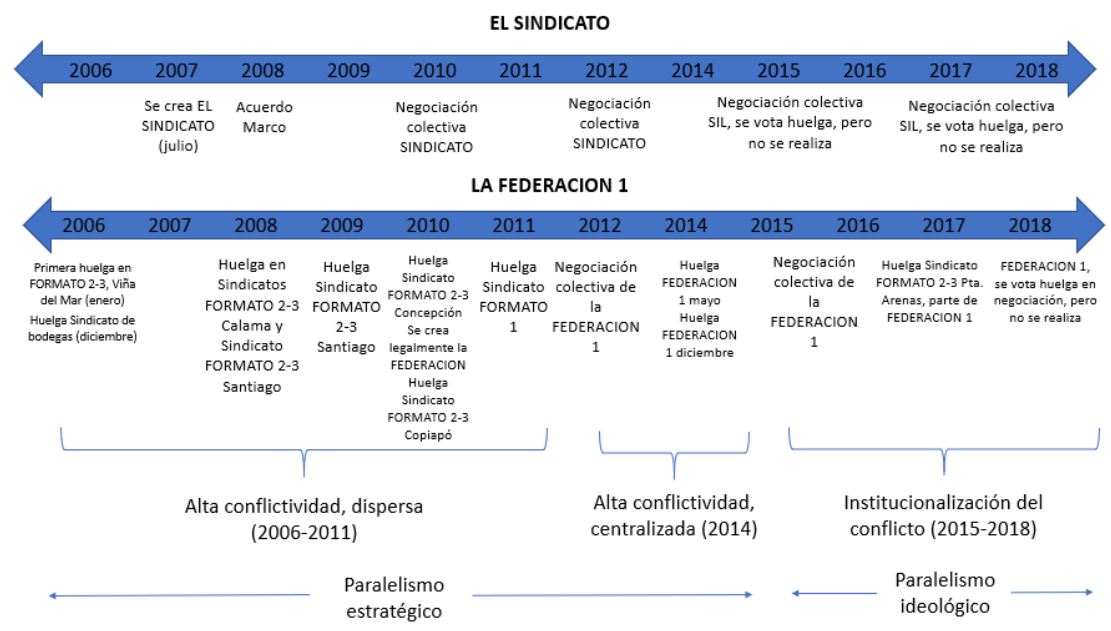

Fuente: elaboración propia en base a revisión de prensa, entrevistas y reportes de sustentabilidad de la empresa.

Pese al paralelismo estratégico entre las dos principales organizaciones de trabajadores, ambas fueron un punto de irrupción y novedad al interior de la empresa, considerando que en el año 2006 la sindicalización abarcaba a solamente 1.500 trabajadores, un $4 \%$ del total de la fuerza de trabajo (Durán y Kremerman, 2007), mientras que en 2016 esta alcanza a un $68,7 \%$ de los 50.762 trabajadores de la empresa (Walmart Chile, 2016:66). Un dirigente del SINDICATO comenta al respecto: "pasamos de una empresa antisindicato a tener más de 130 sindicatos en la compañía". Este aumento exponencial de la sindicalización se ha dado en el marco de un giro radical de la empresa en su disposición hacia los sindicatos: "(buscamos) promover el diálogo y la modernización de las relaciones laborales entre la empresa y los sindicatos en el marco de la reforma laboral" (Walmart Chile, 2016:17), lo que contrasta con la marcada política antisindical de la transnacional a la que pertenece la empresa (Bank, 2017; Gariazzo, 2007; Ubilla, 2011). De hecho, con la llegada del capital transnacional a Chile, en vez de elaborarse una estrategia antisindical, como la que sostuvo la empresa al menos hasta el año 2006, se crea una Gerencia de Relaciones Laborales, que se vincula directamente con los sindicatos y que vela por el cumplimiento de los contratos colectivos. 
Con (la transnacionalización de la empresa) se arma la gerencia de relaciones laborales, tenemos nuestra contraparte ahí. Ellos se preocupan de que se cumpla lo pactado y la Ley. Ellos llevan relación con los sindicatos. Es gente que trabajó en la Dirección del Trabajo, no es gente ingeniera que solo le importan los números. La empresa es muy rigurosa en el cumplimiento de las leyes. La empresa entendió que ya había sindicato, compró sabiendo que había sindicato (Entrevista SINDICATO).

Esta situación de aceptación y puertas abiertas con los sindicatos contrasta con la disposición empresarial antisindical de la empresa a inicios de los 2000:

Cuando se formó este sindicato, yo tenía por lo menos sus 20 cámaras en mi espalda (...) tachaban en un listado todas las personas que me saludaban, todas las personas que se dirigían a mí. (...) el acoso era inmenso po. (...) Cuando se creó este sindicato a los días siguientes llegó gente de la empresa a preguntar por qué se había creado el sindicato. Entonces, yo le dije: “(...) Si usted hubiese tenido respeto por los trabajadores, posiblemente no habría sido innecesario crear un sindicato. Entonces, ustedes han formado esto por los bajos sueldos (...)" (director FEDERACION 1. Video FEDERACION 1).

\section{DEL RÉGIMEN DE TRABAJO “DESPÓTICO” AL “HEGEMÓNICO SEGMENTADO”}

Si se compara la situación en la empresa a inicios de primera década de los años 2000 con la situación de esta a fines de la segunda década del mismo milenio, los cambios son gigantescos. En términos conceptuales se puede argumentar que hubo un cambio en el régimen de producción de la empresa si se comparan los dos tiempos señalados. El régimen de producción de la empresa pasó desde uno "despótico" a uno "hegemónico segmentado", que se expresa en contratos colectivos anuales y bianuales con importantes aumentos salariales y beneficios monetarios especialmente para los trabajadores más antiguos y de los FORMATOS 2 y 3 y, en la disminución de los abusos de las jefaturas de rango medio hacia los trabajadores. Pero este régimen trae consigo elementos que no necesariamente son tan beneficiosos para los trabajadores y que en ocasiones han sido contestados por estos y por los sindicatos. Estos elementos son la institucionalización del conflicto colectivo e individual mediante canales formales para resolver los malestares en la empresa (negociaciones colectivas cada vez más grandes y procedimientos de quejas individuales vía sindicatos), los 
intentos de cooptación normativa de los trabajadores por la cultura de la empresa, la continua intensificación y polifuncionalidad del trabajo, y la presencia de importantes segmentos de "trabajadores precarios": los de FORMATO 4, FORMATO 1 y FORMATO 5; y los trabajadores con menor antigüedad. A continuación, se revisarán los cambios en el régimen de trabajo en distintas dimensiones.

\section{a) Cambios en el proceso de trabajo: mayor intensidad, reglas claras y menos abusos}

Hace diez años o más el trabajo en la empresa era menos intenso y polifuncional. Esto porque había más trabajadores en los locales de trabajo, a quienes se les encomendaban menos funciones; y porque había menos estímulos a la productividad y varios procesos de trabajo aún no eran automatizados. Sobre la cantidad de trabajadores en los locales, un ex vendedor y cajero entrevistado por Abarca (2016) menciona que en los FORMATOS 2 y 3 antes había 600 trabajadores, mientras que ahora hay alrededor de 300. Esto conllevaría que cada trabajador deba más funciones que antes:

Si el cabro tiene que hacer tres pegas está bien, pero págale que sea por dos pegas po, si te estay ahorrando plata. (...) Walmart está creciendo, va a construir 100 locales nuevos, pero no va a contratar más gente po (...) Ahora tienes menos gente, lo que hacían 10 antes ahora lo hacen 2. Por un tema de polifuncionalidad (Entrevista SINDICATO).

Sobre los estímulos a la productividad y el control estricto de los tiempos de trabajo, estos se notan principalmente en el oficio de las cajeras, quienes reciben un "bono por estímulo de ventas", el cual está garantizado en los contratos colectivos de los trabajadores ${ }^{7}$, y a quienes se les mide sistemáticamente el tiempo de trabajo:

Pero a nosotros nos miden todo, el minuto en que tú abres tu caja, el minuto en que empezaste a vender, el minuto en que dejaste de vender, a qué hora cerraste tu caja. $Y$ todo eso es una estadística que te sale diaria, y a fin de mes todo eso queda en el computador y al final de mes tu jefa lo mira y dice "Ah, tal semana cumpliste con un $75 \%$ de lo que se solicita cuando en verdad debieras cumplir con un 80\%" (Entrevista Cajera Maipú FEDERACION 1, por Abarca, 2016). 
El control de los tiempos de trabajo también se ha tornado posible, para todos los trabajadores, por los bonos de "puntualidad" (correcta marcación) y "asistencia" que también garantizan los contratos colectivos del SINDICATO y la FEDERACION 1:

Este incentivo se pagará única y exclusivamente cuando el trabajador cumpla los siguientes requisitos copulativos: (1) cumplir con el 100\% de la asistencia programada efectiva mensual (...), (2) registrar su horario de ingreso y de salida del trabajo en el sistema de control dispuesto, sin dejar de hacerlo (...), e (3) ingresar puntualmente a cumplir sus labores diarias a la hora fijada según los horarios establecidos (Contrato Colectivo FEDERACION 12018. Es similar para el Contrato del SINDICATO del 2017).

Como se observa, el sistema de marcación de la entrada y salida de los trabajadores es fruto de las negociaciones colectivas de las organizaciones sindicales. Estos sistemas fomentan que el trabajador marque la hora de entrada y salida a tiempo, pues si lo hace se gana un bono. Este bono, para los trabajadores con jornada completa, es de $\$ 40.100$ (Contrato Colectivo FEDERACION 1, 2018) y $\$ 34.000$ (Contrato Colectivo SINDICATO, 2017) ${ }^{8}$. Gracias a este nuevo sistema parece ser que a los trabajadores ya no se les deja trabajando después de marcar sus salidas:

Instalamos la política de no vulneración de derechos fundamentales, por lo tanto, no es llegar y tomar arbitrariedades. El trato hacia el personal es muy distinto, tenemos un bono de asistencia y marcación, o sea que si marcas la hora correctamente y te vas te ganas un bono. Con esto se impide que sigas trabajando después de marcar la salida (Entrevista SINDICATO).

Respecto a los malos tratos y los abusos, un dirigente sindical explica que estos fueron problemas del pasado: "Es otra la pelea ahora, el maltrato ya lo superamos. El desafío ahora es la automatización, la polifuncionalidad (...) Hoy hay una política en la empresa distinta" (Entrevista 1 SINDICATO). Según varios entrevistados y fuentes de información, las relaciones entre trabajadores y superiores, como jefes de sección y gerentes de local, se basaban en los malos tratos y en el abuso. Ejemplos de malos tratos son el insultar a los trabajadores o revisar sus pertenencias cuando terminaban sus jornadas. Y ejemplos de abusos son el no pagarles las horas extra y hacerles marcar la salida pero dejarlos trabajando. 
(...) el trato era garabatear a los trabajadores, marcar la salida y dejarlos trabajando igual. Unas arbitrariedades y abusos laborales que son inconcebibles hoy. La gente nueva que llega hoy no se lo imagina. Tenías que llegar con pelo corto, bien afeitado, no permitían tatuaje (...) hoy es una taza de leche, pero antes no podías usar el pelo largo, las cajeras moño, tenías que ir afeitado, no podías usar tatuaje, marcabas salida y seguías trabajando, no había condiciones laborales de seguridad. Había garabatos, para arriba y para abajo (Entrevista SINDICATO).

Que un jefe le revisara la mochila a una trabajadora diciendo que los calzones eran de la marca que se vende en FORMATO 2-3 y que no tenía la boleta y muchas cosas, todas las que se te puedan ocurrir, desde que acusan a un trabajador de robo hasta que un jefe le gritó "hueón" a un trabajador delante de los clientes, de todo lo que pueda suceder, y todo eso fue denunciable y a medida que íbamos ganando denuncias íbamos ganando la confianza de los trabajadores y creciendo la organización sindical (Jorge Salas, en Conteras, 2015).

Aquí en el FORMATO 2-3 la cosa era muy mala en el sentido del trato de los trabajadores, prácticas de patrón de fundo donde se trataba muy mal a la gente y era necesario hacer algo (Entrevista FEDERACION 1).

En los últimos años estas situaciones cambiaron. Hoy en día los malos tratos por parte de los superiores son escasos, y si ocurren, los trabajadores pueden reclamar, ya sea por los canales formales de la empresa o a través los mismos sindicatos. En general tienen éxito al hacerlo:

Hoy se descubre a un jefe dejando a la gente trabajando después del horario, ese jefe se va, ni siquiera amonestación. Un jefe que maltrate o chispee los dedos o te diga un garabato al trabajador ese jefe se va. No podi decirle tonto al trabajador. Claro falta mucho por hacer, la pelea hoy es otra, más sistemática (...) es contra la polifuncionalidad (...) (Entrevista 1 SINDICATO).

\section{b) Cambios en el proceso de trabajo: ¿nuevos aparatos ideológicos?}

Con la llegada del capital norteamericano a Chile (2009) se montó todo un aparataje comunicacional para difundir su cultura organizacional (Ubilla, 2011). Más allá de los eslóganes y los valores (integridad, servicio, respeto, excelencia, entre otros) promovidos por la empresa desde su llegada al país, queda la pregunta de si las actitudes específicas que les exige a sus trabajadores cambiaron o no. Dos actitudes podrían ser candidatas: el grito matinal de las iniciales de la empresa y el intento de que los trabajadores 
se identifiquen como "colaboradores". Como se verá, ambas parecen ser resistidas por los trabajadores, tanto al nivel del lugar de trabajo, de forma individual, como al nivel judicial, mediante estrategias sindicales.

El grito matinal consiste en que "cada día, al empezar la jornada de todos los supermercados, se realiza el grito institucional. No importa el idioma, no importa el país, cada supermercado de la empresa en el mundo comienza su día gritando con fuerza las letras del nombre de la empresa" (Ubilla, 2011). En base a la información revisada, no queda claro si este grito ya existía en la empresa, si se implementó con su transnacionalización o si se intentó implementar, pero no funcionó por las resistencias activas y culturales de los trabajadores. Por ende, no queda claro si es o no un nuevo mecanismo de integración emocional de los trabajadores a la empresa, en el marco de un nuevo régimen de producción hegemónico."Ese cantito de la empresa y que poco menos que hay que adorar al dueño de la empresa, yo no estoy de acuerdo con eso para nada, no me gusta, no comparto su idea" (Entrevista a Secretaria SINDICATO, en Ubilla, 2011).

Todos los días en el supermercado se hace una reunión diaria, que es la reunión donde se dice cómo fue la venta del día anterior, qué es lo que se espera para ese día, se da el grito de la empresa, y se ponen todos y los gritan por letra y todo. Ahí se reúne gente, de repente no está el jefe voy yo, u otro compañero (...) E: ¿Y todos participan del grito? (...) Tienen que participar, porque por algo van ahí, aunque salgas para atrás, porque te retaron dentro de la misma reunión, tienes que quedarte piola. Y así es la cosa (Entrevista a ex vendedor y cajero, por Abarca, 2016).

Otro intento de "control normativo", o de permear con la cultura organizacional de la empresa a los trabajadores, fue el de llamarlos "colaboradores" o "asociados". Esta parece haber sido una estrategia fallida para la compañía, según escribe Bank (2017:124-125), pues los sindicatos "independientes" (las federaciones menores) presentaron una queja en 2010 ante el Ministerio de Trabajo, objetando el término, considerando queel uso de "colaboradores" sería una violación del Código del Trabajo. En 2011 el Ministerio del Trabajo reglamentó que llamar a los trabajadores "colaboradores" efectivamente es una violación al Código del Trabajo (Inspección Comunal del Trabajo Santiago Norte, 2012. Ordenanza $n^{\circ}$ 9), en tanto el concepto jurídico de "trabajador" es el que el Código del Trabajo estableció para referirse a quien presta servicios remunerados 
bajo subordinación y dependencia de otra persona y, porque "induce a error", en tanto coloca al empleador con el colaborador en un plano de horizontalidad ficticia.

\section{c) Las condiciones de empleo se mantienen, pero se modifica el multirut}

En la empresa la flexibilidad por la vía contractual es baja, pero no así la flexibilidad por jornada. Respecto a este último punto, la empresa cuenta con un grupo de alrededor de 20.000 trabajadores con jornadas parciales (menos de 30 horas), los cuáles le permiten ajustar los turnos de trabajo según sus necesidades productivas (principalmente según las afluencias de público). En el Cuadro 3 se presenta la evolución del tipo de contrato y de jornada en la empresa desde el 2007 al 2016; si bien no se cuenta con datos anteriores al 2007, al menos desde ese año hasta el 2016 los cambios son escasos. Aunque la proporción de trabajadores a plazo fijo aumentó en un $10 \%$ en el periodo, el actual $15,5 \%$ sigue estando bajo el porcentaje nacional (27,3\%). Por otro lado, las jornadas laborales permanecen casi idénticas en torno a un $40 \%$. En la empresa la flexibilidad por jornada, como bien ha señalado la literatura sobre el retail en Chile (Díaz et al., 2000), ha estado presente desde los ochenta. La flexibilidad de las jornadas en la empresa parece heredarse desde el régimen despótico al hegemónico segmentado, por lo que no es una característica propia de este último.

Cuadro 3

Proporción de trabajadores en la empresa según contrato y jornada laboral en la empresa (2007-2017)

\begin{tabular}{lccccc}
\hline & $\mathbf{2 0 0 7}$ & $\mathbf{2 0 0 8}$ & $\mathbf{2 0 0 9}$ & $\mathbf{2 0 1 6}$ & $\mathbf{2 0 1 7}$ \\
\hline Plazo fijo & $6 \%$ & $17,60 \%$ & $13,30 \%$ & $16,60 \%$ & $15,50 \%$ \\
\hline Plazo indefinido & $94 \%$ & $82,40 \%$ & $86,70 \%$ & $83,40 \%$ & $84,50 \%$ \\
\hline Jornada completa & $58,10 \%$ & $57,30 \%$ & $57,10 \%$ & $61 \%$ & $59,44 \%$ \\
\hline Jornada parcial & $41,90 \%$ & $42,70 \%$ & $42,90 \%$ & $39 \%$ & $40,56 \%$ \\
\hline
\end{tabular}

Fuente: Elaboración propia en base a datos presentados en los Reportes anuales de la empresa (2008-2009; 2016; 2017).

La existencia de cinco grandes formatos, en donde cada uno corresponde a un RUT particular (definición legal de empresa), es un cambio reciente realizado por la empresa (2012-2013). Antes de que existieran los cinco grandes formatos-empresas eran más de 200 los RUT que contrataban a los trabajadores, lo que dificultaba enormemente la 
organización sindical y la negociación colectiva: "En la empresa eran 200 razones sociales distintas, cada local era una razón social distinta" (Entrevista SINDICATO).

Si bien en Chile hasta los años 2000 ocurrió una desregulación e intensificación del trabajo en el sector del comercio (Díaz, Ruiz Tagle, Aguilar, y Frías, 2000; Gálvez, Henríquez, y Morales, 2009), desde mediados de la misma década la intensificación del trabajo ha sido progresivamente regulada desde el Estado, ante la creciente actividad política (lobby) de los sindicatos del sector. Así emerge la Ley 19.759 de 2001 que entra en vigor recién en 2005- que reduce de 48 a 45 horas semanales la jornada de trabajo en el país; la Ley 20.281 de 2008 que establece que el sueldo base de los trabajadores que reciben comisiones por venta o productividad (y que trabajan jornada completa) no podrá ser inferior a un ingreso mínimo mensual; la Ley 20.760 de 2014 o Ley "multirut" que considera como un solo empleador para efectos laborales y previsionales a dos o más empresas cuando tienen una dirección laboral común o se prueba la existencia entre ellas de un controlador común; y la Ley 20.833 del 2015, que establece que en el sector comercio las horas ordinarias trabajadas en día domingo deberán ser remuneradas con un recargo de, al menos, un 30\%, calculado sobre el sueldo convenido para la jornada ordinaria.

Pese a que en el año 2014 se aprobó la Ley 20.760 que regula el multirut de las empresas, el cambio de 200 a 5 razones sociales por parte de la compañía fue un proceso que se dio antes de la aprobación de la Ley. Aparentemente, la empresa vio venir esta nueva Ley, en tanto las discusiones parlamentarias comenzaron en 2008 y los sindicatos de la empresa participaron activamente en estas discusiones. Si surgía una Ley para regular el multirut y al interior de la empresa ya existían dos organizaciones que vía figuras legales como el "sindicato interempresas" o "federación" habían superado las divisiones entre RUT, no tenía sentido seguir insistiendo con la ultra división jurídica de la compañía. Tras la simplificación de las razones sociales, en el año 2012 el SINDICATO negocia de forma reglada con la empresa, alcanzando un contrato colectivo para el FORMATO 3 y otro para el FORMATO 2. Así también, en el año 2014 la FEDERACION 1 negocia con la empresa y firma 3 contratos colectivos: uno para FORMATO 2, otro para FORMATO 3 y otro para FORMATO 4. Pero no es hasta 2015 que verdaderamente se le gana la pelea del multirut, cuando en base a la Ley 20.760 se llega a un acuerdo vía demanda judicial. Es 
este año cuando el SINDICATO, y el que sigue la FEDERACION 1, logran firmar contratos colectivos únicos para cada organización, que involucran a las cinco razones sociales en un solo contrato colectivo.

Cuando llega la empresa norteamericana se adelanta un poco a esto que se veía venir del fin al multirut. Unifica sus razones sociales en FORMATO 2, FORMATO 3, FORMATO 4 y FORMATO 1. Ahora eran cinco razones sociales, pero igual teníamos que firmar cinco contratos. A partir del multirut, que ganamos un juicio a la empresa, esta reconoce que la empresa es la misma para los cinco supermercados. (...) supermercadista son cinco razones sociales y ahora firmamos solamente un contrato colectivo (Entrevista SINDICATO).

Esta forma de negociación, que se mantiene hasta ahora, implica un gran contrato colectivo para cada una de las grandes organizaciones sindicales. El problema de este sistema, como se verá en el siguiente apartado, es que cada uno de los cinco formatos tiene clausulas específicas en el contrato colectivo, las cuales son altamente desiguales en relación con los beneficios materiales que reciben los trabajadores de cada formato.

\section{d) Cambios en el soporte material: segmentación de la seguridad salarial y de los} incentivos

Respecto al soporte material, es importante partir del el hallazgo de que el salario mínimo del país es igual al que entrega la empresa. Es decir, hoy debería estar en $\$ 301.000$ (desde marzo de 2019). Sin embargo, este monto se ve incrementado por una serie de beneficios e incentivos, los cuales han aumentado crecientemente en la empresa, especialmente para los trabajadores más antiguos y los de los FORMATOS 2-3. Estos incrementos salariales, tanto por beneficios como por incentivos, son entregados de forma impersonal a los trabajadores sindicalizados, a través de contratos colectivos muy similares entre las distintas organizaciones sindicales. Dentro de estos beneficios una parte importante es variable, la cual se entrega según el buen comportamiento de los trabajadores (marcar bien y a tiempo la salida y entrada) y su productividad (bono estímulo de ventas, para el caso de las cajeras); y la otra parte es constante (bono de antigüedad, reajuste, bono de movilidad, aguinaldos, etcétera), basada en beneficios que no dependen del desempeño del trabajador, sólo de su antigüedad y del formato en el que trabaja. 
Para el año 2017-2018 los beneficios garantizados por los contratos colectivos firmados por el SINDICATO y la FEDERACION 1 son los siguientes: Bono Fijo Mensual (FORMATOS 2-3 de la FEDERACION 1), Bono Trabajador del SINDICATO anual (todos los formatos del SINDICATO), Reajuste general de salario (todos los formatos SINDICATO y algunos FEDERACION 1), Premio al mejor colaborador del año, Fiesta de Navidad y regalo de navidad para los hijos, posibilidad de hacer la Nivelación de estudios (y sacar el cuarto medio), Seguro médico, Seguro de vida, Convenio dental, Certificación de habilidades y posibilidad de ascenso interno (sólo SINDICATO), Fin de semana para dos como premio de reconocimiento por la antigüedad del trabajador, Seguro de trabajadores por muerte o invalidez, Sala Cuna Garantizada, Bonos y permisos por Fallecimiento de cónyuge o hijo, y de padre o madre, Permisos por muerte de hermanos o abuelos, Becas anuales de estudio para hijos con excelencia académica, Préstamo marzo, Gratificación anual de 4,75 sueldos mínimos para jornadas de 45 horas, Bono estímulo de caja (0,18\% para SINDICATO y 0,19\% para FEDERACION 1 por cada millón), Asignación por pérdida de cajas, Bono para Tesorero/supervisor de cajas, Bono por resultados operacionales (ROP), Ropa e implementos para los indefinidos (a mejor formato, mejores y más implementos), Incentivo asistencia y de correcto registro de ingreso y salida, Bono Movilización por día trabajado, Buses de acercamiento ala empresa en circunstancias especiales (solo FEDERACION 1 en FORMATO 2-3), Aguinaldo Fiestas Patrias, Bono por uso de vacaciones en temporada alta (FORMATO 2-3), Incentivo por trabajar el día de la madre, Semana Santa, fiestas patrias o navidad, Bono por trabajar en Jornada nocturna, Bono por nacimiento o adopción, Permiso y bono por matrimonio, Torta de Cumpleaños, Descuento y premiación por comprar en el supermercado, Préstamo de vivienda (FORMATO 2-3), Prestamo especial (FORMATO 2-3), Almuerzo (FORMATO 2-3) y Bono de Término de Negociación (BTN).

Como se observa, son muchísimos los beneficios. A modo de resumen y como interpretación general, se puede decir que entre los beneficios obtenidos por el SINDICATO en 2017 y la FEDERACION 1 en 2018 hay pequeñas diferencias. Las diferencias más importantes, tanto por la cantidad de beneficios, como por la magnitud de los mismos, de dan entre los trabajadores de los diferentes formatos y entre los trabajadores de diferentes antigüedades. A continuación, se presentara el análisis de las diferencias en uno de los beneficios más importantes: el Bono de término de conflicto (BTN). Lo que sucede con este bono sucede prácticamente con todos los otros beneficios de los contratos colectivos. 
Los bonos de término de negociación (BTN) son un beneficio exclusivamente sindical que, al menos hasta el año 2008, escasos trabajadores recibían. En las últimas negociaciones del SINDICATO y de la FEDERACION 1, este bono ha alcanzado cifras muy similares (ver Cuadro 4), aunque muy desiguales según la antigüedad de los trabajadores: quienes tienen una antigüedad de más de seis años en la empresa y pertenecen a los FORMATOS 2-3 recibieron más de un millón de pesos (más de tres veces el sueldo mínimo), mientras quienes tienen una antigüedad menor a un año reciben $\$ 180.000$ o menos (alrededor de la mitad de un sueldo mínimo).

\section{Cuadro 4}

Bonos de término de negociación (BTN) del SINDICATO (2017) y la FEDERACION 1 (2018) para los formatos FORMATO 2-3

\begin{tabular}{|c|c|c|}
\hline Antigüedad de los trabajadores & FEDERACION 1 & SINDICATO \\
\hline Menos de 6 meses & 110.000 & 100.000 \\
\hline Entre 6 meses - 1 año & 160.000 & 180.000 \\
\hline Entre 1 a 2 años & 320.000 & 350.000 \\
\hline Entre 2 a 3 & 595.000 & 630.000 \\
\hline Entre 3 a 4 & 640.000 & 680.000 \\
\hline Entre 4 a 5 & 780.000 & 780.000 \\
\hline Entre 5 a 6 & 810.000 & 800.000 \\
\hline Entre 6 a 7 & 1.000 .000 & 1.000 .000 \\
\hline Entre 7 a 8 & 1.110 .000 & 1.100 .000 \\
\hline Entre 8 a 9 & 1.210 .000 & 1.200 .000 \\
\hline Entre 9 a 10 & 1.390 .000 & 1.380 .000 \\
\hline Entre 10 a 11 & 1.570 .000 & 1.550 .000 \\
\hline Entre 11 a 12 & 1.620 .000 & 1.600 .000 \\
\hline Entre 12 a 13 & 1.670 .000 & 1.650 .000 \\
\hline Entre 13 a 14 & 1.690 .000 & 1.650 .000 \\
\hline Entre 14 a 15 & 1.810 .000 & 1.800 .000 \\
\hline Entre 15 a 16 & 1.830 .000 & 1.800 .000 \\
\hline Entre 16 a 17 & 1.870 .000 & 1.800 .000 \\
\hline Entre $17 \mathrm{y}$ mas & 1.960 .000 & 1.800 .000 \\
\hline
\end{tabular}


Esta diferencia del BTN entre trabajadores con distinta antigüedad también se replica entre los formatos. Mientras el trabajador recién llegado al FORMATO 1 recibe un BTN de $\$ 55.000$ (FEDERACION 1) o $\$ 60.000$ (SINDICATO), el trabajador recién llegado al FORMATO 2-3 obtiene un bono de casi el doble: $\$ 110.000$ (FEDERACION 1) o $\$ 100.000$ (SINDICATO). En el otro extremo, un trabajador de FORMATO 2-3 con la mayor antigüedad recibe un BTN de $\$ 1.960 .000$ (FEDERACION 1) o $\$ 1.800 .000$ (SINDICATO), mientras que uno de la mayor antigüedad del FORMATO 1 o FORMATO 5 solamente recibe $\$ 210.000$ (FEDERACION 1) o \$200.000 (SINDICATO), es decir, casi un décimo de lo que recibe el trabajador del FORMATO 2-3.

Cuadro 5

Diferencias por formato en los BTN obtenidos por SINDICATO (2017) y FEDERACION 1 (2018) según antigüedad

\begin{tabular}{|c|c|c|c|c|}
\hline $\begin{array}{l}\text { Antigüedad / } \\
\text { Formato }\end{array}$ & FORMATO 2-3 & FORMATO 4 & FORMATO 5 & FORMATO 1 \\
\hline $\begin{array}{l}\text { BTN para la } \\
\text { menor antigüedad }\end{array}$ & $\begin{array}{l}110.000 \\
\text { (FEDERACION 1) } \\
\text { y } 100.000 \\
\text { (SINDICATO) }\end{array}$ & $\begin{array}{l}65.000 \\
\text { (FEDERACION } 1 \\
\text { y SINDICATO) }\end{array}$ & $\begin{array}{l}55.000 \\
\text { (FEDERACION 1) } \\
\text { y } 80.000 \\
\text { (SINDICATO) }\end{array}$ & $\begin{array}{l}55.000 \\
\text { (FEDERACION 1) } \\
\text { y } 60.000 \\
\text { (SINDICATO) }\end{array}$ \\
\hline $\begin{array}{l}\text { BTN para la may } \\
\text { antigüedad }\end{array}$ & $\begin{array}{l}1.960 .000 \\
\text { (FEDERACION1) } \\
\text { y } 1.800 .000 \\
\text { (SINDICATO) }\end{array}$ & $\begin{array}{l}540.000 \\
\text { (FEDERACION } 1 \\
\text { y SINDICATO) }\end{array}$ & $\begin{array}{l}210.000 \\
\text { (FEDERACION 1) } \\
\text { y } 200.000 \\
\text { (SINDICATO) }\end{array}$ & $\begin{array}{l}210.000 \\
\text { (FEDERACION } 1 \\
\text { y SINDICATO) }\end{array}$ \\
\hline
\end{tabular}

Fuente: elaboración propia en base a revisión de contratos colectivos.

Los beneficios del SINDICATO y la FEDERACION 1, asegurados vía contratos colectivos, han crecido progresivamente desde sus primeras negociaciones no regladas (SINDICATO) o descentralizadas (FEDERACION 1) y cada vez han tendido a parecerse más entre sí. Las cláusulas innovadoras y exitosas de cada contrato son copiadas por los otros sindicatos y los beneficios extendidos a los trabajadores no sindicalizados, por lo que todas las victorias sindicales expresadas en los contratos colectivos han pasado a ser "políticas de la empresa".

La red de beneficios materiales asegurados por los contratos colectivos contrasta con los que obtenían los trabajadores cuando imperaba el régimen de trabajo despótico en la empresa. En ese entonces, los aumentos salariales se realizaban vía negociación individual de cada trabajador con sus superiores. En base al desempeño del trabajador (y 
posiblemente en base a la cercanía con el superior) se le subía el sueldo. Con el cambio de régimen se ha transformado la forma mediante la cual la empresa le otorga beneficios monetarios y sociales a sus trabajadores: ya no lo hace de manera personalizada en base al desempeño individual, sino mediante contratos colectivos muy similares entre las distintas organizaciones sindicales. Si un trabajador actualmente quiere aumentar su salario, tener beneficios, o incluso en ocasiones gestionar cambios de jornada, deberá sindicalizarse:

Antes si un trabajador del área de más bajo sueldo tenía posibilidades de obtener un aumento de sueldo, iba, lo conversaba, si cumplía con el horario y todas las metas de trabajo, y el jefe estaba de acuerdo, el jefe iba lo conversaba y le subían el sueldo. Hoy día no se sube el sueldo si a toda la gente no se le sube (...) Tú no tienes la posibilidad de destacarte entre medio de tus pares en forma económica (...) la mayoría de la gente se ha sindicalizado, porque es la única forma de obtener beneficios económicos es a través de los sindicatos, de las negociaciones. Hoy en día cada dos años están negociando los sindicatos y ellos obtienen por la negociación por término de conflicto de $600 \mathrm{mil}$ o $700 \mathrm{mil}$, depende de los años de trabajo que se tenga (Entrevista a ex vendedor y cajero, por Abarca, 2016).

\section{DISCUSIÓN Y CONCLUSIONES}

Tras décadas de silencio, el trabajo precario en el retail neoliberal chileno ha generado sus propios contramovimientos entre los trabajadores y trabajadoras. En el presente artículo se ha descrito el proceso de emergencia de organizaciones sindicales desde mediados de los años dos mil en la empresa Walmart Chile, los que modificaron radicalmente el panorama de las relaciones laborales en la empresa: esta pasó de tener una escasa y débil presencia sindical (4\% de afiliación), en el marco de una política antisindical, a una alta presencia sindical (más del 70\% de afiliación), en el marco de una renovada política de modernización y diálogo social. Esta alta tasa de sindicalización, por efecto de la legislación chilena que lo permite, se encuentra dividida en dos grandes organizaciones sindicales altamente representativas: el Sindicato Interempresas de Trabajadores de Líder y la Federación del Trabajador de Walmart, los que en los últimos diez años han desarrollado estrategias diversas que han tendido hacia la convergencia y la moderación de la disrupción sobre la producción. 
La presión "desde abajo" de los sindicatos y "desde arriba" de las regulaciones nacionales (como la Ley del multirut de 2015), en el marco de una transnacionalización y un crecimiento económico sostenido de la empresa, han promovido un cambio en el régimen de producción. Es decir, no solo la organización sindical emerge como respuesta a la precarización del trabajo, como han señalado largamente los estudios de la revitalización sindical (Frege y Kelly, 2004), los recursos de poder (Fichter et al., 2018) y la precariedad del trabajo (Atzeni y Ness, 2018), sino ésta se vuelve un agente activo en su transformación. Es decir, la precarización del trabajo no es un condición estática (Ikeler, 2019), ni la mercantilización de la sociedad una tendencia de todos los frentes, progresiva e irreversible. La organización y presión obrera promueven la reestructuración del capital y la modificación de sus regímenes, destruyendo las viejas condiciones materiales desde las cuales los trabajadores se organizaban (Campbell, 2018). El cambio en el régimen de trabajo, lejos de haber sido producto de la ausencia de poder obrero y de la pura voluntad empresarial, fue consecuencia de la lucha de clases al interior de la empresa estudiada. Se deberán seguir probando estas hipótesis sobre la capacidad estructurante de la acción sindical, sobre la mutabilidad de las dominaciones del capital (de sus regímenes) y sobre las posibilidades de "desprecarización" del trabajo en otros sectores económicos y países. Estos aspectos deberían considerarse como elementos claves a la hora de evaluar los efectos y orientaciones de los recursos de poder de la clase trabajadora (Nowak, 2018).

Se ha mostrado que el régimen de trabajo en Walmart Chile pasó de uno "despótico" basado en la desprotección legal, la inseguridad salarial, el abuso y las prácticas antisindicales, a otro "hegemónico segmentado", con mayores protecciones legales, seguridad salarial diferenciada, buen trato, intentos de control normativo y respeto de la sindicalización. En este nuevo régimen los trabajadores han conseguido una serie de beneficios materiales por sobre el sueldo mínimo, mediante negociaciones colectivas periódicas bianuales; han resistido algunos intentos de control normativo de la empresa; son agentes activos a la hora de fiscalizar los actos discriminatorios y abusivos de los mandos medios y de las gerencias ${ }^{9}$ y han sido actores claves a la hora de regular y valorizar los tiempos en el trabajo a partir de cláusulas específicas de los contratos colectivos (entrada, salida, bono de cajeras, entre otros).

Todos los cambios mencionados parecen ser concesiones $\mathrm{u}$ omisiones "beneficiosas" por parte de la empresa hacia sus trabajadores, pero pese a estos cambios, ésta no ha visto perjudicados sus intereses: ha seguido 
creciendo en ventas, en número de trabajadores y en el porcentaje utilizado del mercado supermercadista (Walmart Chile, 2016). Es más, ha podido seguir intensificando el trabajo en base a la polifuncionalidad de cada trabajador y en base a un sistema de turnos flexibles, ajustado a la demanda, que las cuatro décimas partes de los trabajadores hacen posible: los con jornada parcial. Además, si bien ha tenido que ceder una serie de beneficios materiales, como millonarios bonos de términos de conflictos o ajustes de sueldos por sobre el $\mathrm{IPC}^{10}$, lo ha hecho a cambio del cese de las disrupciones sobre la producción ${ }^{11}$ y la "sobreexplotación" de un segmento de los trabajadores de la empresa: los más nuevos y los de los formatos de trabajo más precarios, a quienes les llegan beneficios materiales mucho más bajos que a los trabajadores de mayor antigüedad y a los de formatos salarialmente seguros. Frente esta situación, uno de los principales desafíos de los sindicatos de la empresa va en la línea de superar la comentada dualización (Visser, 2019).

De esta forma, se va esclareciendo el "intercambio" o "compromiso positivo" (Wright, 2000) en el que se encuentran los trabajadores y la empresa en el actual régimen de producción "hegemónico segmentado": se consiente la intensificación del trabajo y la segmentación de los beneficios materiales a cambio del reconocimiento sindical, los aumentos salariales directos e indirectos para un segmento de trabajadores y la reducción de los malos tratos, los abusos y las discriminaciones hacia los trabajadores. En resumen, hay una "intensificación negociada", una especie de negociación del esfuerzo (Edwards, 1990) que va más allá del proceso de trabajo y que le permite a la empresa mantener y aumentar sus ganancias sin perder el control del trabajo ante el fracaso de la política anti sindical y de despotismo de mercado.

Una de las grandes enseñanzas que le ha dejado a los estudiosos del trabajo la irrupción del neoliberalismo desde fines del siglo XX es que ningún compromiso de clase, ya sea a nivel estatal (Dörre et al., 2009) o a nivel de empresa (Burawoy, 2014) es eterno. Apenas la clase capitalista ha tenido la alternativa de avanzar en sus intereses y lo ha hecho, presionando o directamente rompiendo todos los acuerdos que ha alcanzado con sus contrapartes. Los estudiosos del trabajo y los actores sindicales no debemos darle la espalda al futuro, pues quedarse ensimismado en las grandes transformaciones de los últimos años puede hacer que pasen inadvertidas las nuevas tendencias que eventualmente pueden provocar el quiebre del actual régimen de producción. Esto, por los efectos que podrían provocar los procesos de 
automatización internos; las revueltas populares de octubre de 2019 y su prolongación institucional en el debate constituyente; o el coronavirus y el aislamiento social. El riesgo de una eventual precarización "no consentida" en la empresa está a la vuelta de la esquina.

(Recibido el 25 de mayo de 2019)

(Reenviado el 12 de abril de 2020)

(Aprobado para su publicación el 17 de mayo de 2020)

\section{NOTAS}

1. Para una revisión de las diferentes olas de las teorías del proceso de trabajo véase Thompson (2010) y/o Castillo y Ratto (2018), quienes realizan una síntesis en español de esta discusión.

2. La preocupación de Burawoy no va tanto en la línea de explicar los estallidos ocasionales de resistencia, o los niveles de ausentismo y las restricciones a la producción, sino el hecho de que los trabajadores recurran cotidianamente a la fábrica a explotarse, aún contra su propio beneficio.

3. Junto a las otras dos grandes empresas que le siguen en tamaño controlan el $80 \%$ del mercado (Feller Rate, 2013).

4. Este está "focalizado en ventas al por mayor, atiende exclusivamente a comerciantes asociados mediante un sistema de membresía gratuita" (Walmart Chile, 2016).

5. Según un dirigente entrevistado, este formato es el que más se parece en Chile al clásico local de la empresa en Los Estados Unidos. La empresa estaría buscando desarrollar con más fuerzas este formato, en tanto le genera una alta ganancia y le implica bajos niveles de inversión.

6. Con este la empresa "busca estar cerca del cliente, con precios bajos siempre y para las compras del día a día" (Walmart Chile, 2016), casi como un "local de barrio".

7. “ $0,19 \%$ brutos por cada millón y fracción de venta registrada en la máquina registradora asignada (...)" (Contrato Colectivo FEDERACION 1 2018. Es similar para el Contrato del SINDICATO del 2017)

8. Cerca de un $15 \%$ del Ingreso Mínimo Mensual que ganan los trabajadores.

9. La importancia de estas últimas modificaciones radica en que la demanda por un "buena trato", al nivel interaccional, ya sea en el trabajo como en otros ámbitos, es una de las demandas de justicia más sentidas por los trabajadores y trabajadoras en Chile.

10. Índice de Precios al Consumidor. Es una medida de la inflación en el país.

11. La última huelga nacional fue el 2014, liderada por la FEDERACION 1 . Por el otro lado, el SINDICATO nunca ha hecho huelgas. Sorpresivamente el año 2019 el Sindicato realizó una huelga, cuyas motivaciones se discuten en: http://www.revistarosa.cl/2019/07/01/ sobre-la-primera-huelga-del-sil-el-sindicato-mas-grande-de-chile-breve-historia-del-conflicto-y-el-consenso-en-walmart/ 


\section{REFERENCIAS}

ABARCA, Manuela. (2016), Relación entre Trabajo y Salud: Significados y Prácticas en Trabajadores de Supermercados. Tese (Graduação em Sociologia), Universidad de Chile, Santiago.

ANNER, Mark. (2015), "Labor Control Regimes and Worker Resistance in Global Supply Chains". Labor History, vol. 56, n³ 3, pp. 292-307.

ATZENI, Maurizio; NESS, Immanuel (eds.). (2018), Global Perspectives on Workers' and Labour Organizations. London, Springer.

BANK, Carolina. (2017). Building Power from Below: Chilean Workers Take On Walmart. New York, Cornell University Press.

; KEENNEY, Bridget; STECHER, Antonio. (2018), Walmart in the Global South: Workplace Culture, Labor Politics, and Supply Chains. Austin, University of Texas Press.

BRAVERMAN, Harry. (1974), Labor and Monopoly Capital: The Degradation of Work in the Twentieth Century. New York, NYU Press.

BROOKES, Marissa. (2013), "Varieties of Power in Transnational Labor Alliances: An Analysis of Workers' Structural, Institutional and Coalitional Power in the Global Economy". Labor Studies Journal, vol. 38, n 3, pp. 181-200.

BURAWOY, Michael. (1978), "Toward a Marxist Theory of the Labor Process: Braverman and Beyond". Politics y Society, vol. 8, n³-4, pp. 247-312.

. (1983), "Between the Labor Process and the State: The Changing Face of Factory Regimes under Advanced Capitalism". American Sociological Review, vol. 48, $\mathrm{n}^{\circ}$ 5, pp. 587-605.

. (1985), The Politics of Production: Factory Regimes under Capitalism and Socialism. London, Verso Books.

. (2014), "Una Nueva Aproximación a Manufacturing Consent (Manufactura Consensuada)". Revista de Trabajo, $\mathrm{n}^{\circ} 12$, pp. 159-172

CAMPBELL, Stephen. (2018), Border Capitalism, Disrupted: Precarity and Struggle in a Southeast Asian Industrial Zone. New York, Cornell University Press.

CASTILLO, Alejandro; RATTO, Nicolás. (2018), Teorías del Proceso de Trabajo. Una Revisión de su Desarrollo y de las Nociones de Control y Resistencia (Documento de Trabajo $\mathrm{n}^{\circ} 11$ ). Fondecyt 1150860 y CIPSTRA.

CHAN, Jenny; NAIR, Manjusha; RHOMBERG, Chris. (2019), "Precarization and Labor Resistance: Canada, the USA, India and China". Critical Sociology, vol. 45, n 4-5, pp. 469-483.

COLLER, Xavier. (2000), Estudios de Caso. Madrid, Centro de Investigaciones Sociológicas.

CONTRERAS, Pamela. (2015), Estudio Descriptivo del Proceso de Organización Sindical de los Trabajadores de Retail Supermercadista Afiliados al Sindicato Líder n 40 de Valparaíso. Tese (Graduação em Antropologia), Universidad Austral de Chile, Valdivia. 
CURLEY, Caitriona; ROYLE, Tony. (2013), "The Degradation of Work and the end of the Skilled Emotion Worker at Aer Lingus: Is it all Trolley Dollies Now?" Work, Employment and Society, vol. 72, $\mathrm{n}^{\circ}$ 1, pp. 105-121.

DE LA GARZA, Enrique. (2000), “La Flexibilidad del Trabajo en América Latina”. In: E. de la Garza, Tratado Latinoamericano de Sociología del Trabajo, México, Fondo de Cultura Económica, pp. 148-178.

(2009), "Hacia un Concepto Ampliado de Trabajo”. In: J. C. Neffa; E. de la Garza y L. Terra (comp.), Trabajo, Empleo, Calificaciones Profesionales, Relaciones de Trabajo e Identidades Laborales, vol. I, Buenos Aires, CAICCyT CLACSO, pp. 111-140.

DÍAZ, Estrella; RUIZ TAGLE, Jaime; AGUILAR, Omar; FRÍAS, Patricio. (2000), Jornada de Trabajo en el Sector Comercio: La Experiencia en Grandes Tiendas, Supermercados y Pequeñas Tiendas en Malls. Cuadernos de Investigación n ${ }^{\circ}$ 12, Santiago de Chile, Dirección del Trabajo.

DÖRRE, Klaus; HOLST, Hajo; NACHTWEY, Oliver. (2009). “Organising-A Strategic Option for Trade Union Renewal?" International Journal of Action Research, vol. 5, nº 1, pp. 33-67.

DURÁN, Gonzalo; KREMERMAN, Marco. (2007). Informe Retail: Capítulo Retail. Fundación SOL.

EDWARDS, Paul. (1990), El Conflicto en el Trabajo: Un Análisis Materialista de las Relaciones Laborales en la Empresa. Madrid, Ministerio de Trabajo y Seguridad Social, Centro de Publicaciones.

FELLER RATE. (2009). Informe Clasificación de Riesgo DyS. Disponible en: http:/ / www.feller-rate.cl/general2/corporaciones/dys0910.pdf

FICHTER, Michael; LUDWIG, Carmen; SCHMALZ, Stefan; SCHULZ, Bastian; STEINFELDT, Hannah. (2018), The Transformation of Organised Labour. Mobilising Power Resources to Confront 21st Century Capitalism. Berlin, Friedrich-Ebert-Stiftung.

FREGE, Carola; KELLY, John. (2004), Varieties of Unionism: Strategies for Union Revitalization in a Globalizing Economy. Oxford, Oxford University Press on Demand.

FRIEDMAN, Andy. (1977), "Responsible Autonomy versus Direct Control over the Labour Process". Capital \& Class, vol. 1, nº 1, pp. 43-57.

GÁLVEZ, Telma; HENRÍQUEZ, Helia; MORALES, Gabriela. (2009). Holding y Tiendas: La Trama que Multiplica las Ventas y Redobla el Trabajo. Santiago, Dirección del TrabajoGobierno de Chile.

GARIAZZO, Alicia. (2007), La Industria del Retail en Chile y el Modelo Walmart. Cámara de Diputados. Disponible en: https:/ / es.scribd.com/document/174340816/La-Industria-Del-Retail-en-Chile-Alicia-Gariazzo

HARVEY, David. (2007), Breve Historia del Neoliberalismo. Madrid, AKAL.

IBSEN, Christian Lyhne y TAPIA, Maite. (2017), “Trade Union Revitalisation: Where are We Now? Where to Next?". Journal of Industrial Relations, vol. 59, n 2, pp. 170-191.

IKELER, Peter. (2016), “Deskilling Emotional Labour: Evidence from Department Store Retail". Work, Employment and Society, vol. 30, n 6, pp. 966-983.

(2019), "Precarity's Prospect: Contingent Control and Union Renewal in the Retail Sector". Critical Sociology, vol. 45, no 4-5, pp. 501-516. 
KELLY, John. (2012), Rethinking Industrial Relations: Mobilisation, Collectivism and Long Waves. London, Routledge.

KENNY, Bridget. (2018), "Labour Politics and South African Retail Workers: Enduring Collectivities in the Face of Precariousness". In: M. Atzeni y I. Ness (eds.), Global Perspectives on Workers' and Labour Organizations. Singapore, Springer, pp. 85-104.

LÉVESQUE, Christian; MURRAY, Gregor. (2010), “Understanding Union Power: Resources and Capabilities for Renewing Union Capacity". Transfer: European Review of Labour and Research, vol. 16, nº 3, pp. 333-350.

MARKS, Abigail; THOMPSON, Paul. (2010),"Beyond the Blank Slate: Identities and Interests at Work". In: P. Thompson y C. Smith (eds.), Working Life: Renewing Labour Process Analysis (Critical Perspectives on Work and Employment). Palgrave Macmillan.

NICHOLS, Theo; CAM, Surhan; CHOU, Wen-chi Grace; CHUN, Soonok; ZHAO, Wei; FENG, Tongqing Feng. (2004), "Factory Regimes and the Dismantling of Established Labour in Asia: A Review of Cases from Large Manufacturing Plants in China, South Korea and Taiwan". Work, Employment and Society, vol. 18, n 4, pp. 663-685.

NOWAK, JJörg. (2018),"The Spectre of Social Democracy: A Symptomatic Reading of the Power Resources Approach". Global Labour Journal, vol. 9, n 3, pp. 353-360.

RATTO, Nicolás. (2019), “Estrategias Sindicales y Recursos de Poder. Presentación y Prueba Empírica de un Marco para el Análisis de las Variedades Sindicales en Chile y en América Latina". Revista Astrolabio, n 23, pp. 134-168.

RAYO, Karla Fabiola. (2007). Análisis de las Estrategias Comerciales Realizadas por Supermercados Lider en el Período 2000-2006 Tese (Gradução em Engenharia Civil Industrial), Universidad de Chile, Santiago.

SILVER, Beverly J. (2003), Forces of Labor: Workers' Movements and Globalization since 1870. New York, Cambridge University Press.

STECHER, Antonio y MARTINIC, Rodolfo. (2018), "La Descualificación del Trabajo en Tiendas por Departamento". Psicoperspectivas, vol. 17, n³, pp. 40-51.

TATTERSALL, Aamanda. (2013), Power in Coalition: Strategies for Strong Unions and Social Change. New York, Cornell University Press.

TAYLOR, Phil; MOORE, Sian. (2015), "Cabin Crew Collectivism: Labour Process and the Roots of Mobilization". Work, Employment and Society, vol. 29, n 1, pp. 79-98.

THOMPSON, Paul. (2010), "The Capitalist Labour Process: Concepts and Connections". Capital y Class, vol. 34, n ${ }^{\circ} 1$, pp. 7-14.

THOMPSON, Phil; BROEK, Diane van den. (2010), "Managerial Control and Workplace Regimes: An Introduction". Work, Employment and Society, vol. 24, nº 3, pp. 1-12.

UBILLA, Carolina. (2011), Los Procesos de Comunicación Interna en D\&S tras la Llegada de Walmart. Tese (Graduação em Jornalismo). Universidad de Chile, Santiago. Disponível em: http:/ / repositorio.uchile.cl/handle/2250/135188

VISSER, Jelle. (2019), Trade Unions in the Balance. Geneva, International Labour Organization's Bureau for Workers' Activities (ACTRAV). 
Nicolas Ratto

WALMART CHILE. (2016). Reporte de Sustentabilidad 2016.

(2017). Reporte de Sustentabilidad 2017.

WEBSTER, Edward; O’BRIEN, Robert. (2020), “Ten Years of the Global Labour Journal: Reflecting on the Rise of the New Global Labour Studies". Global Labour Journal, vol. $11, \mathrm{n}^{\circ} 1$, pp. $4-17$.

WRIGHT, Erik Olin. (2000), “Working-Class Power, Capitalist-Class Interests and Class Compromise". American Journal of Sociology, vol. 105, $\mathrm{n}^{\circ} 4$, pp. 957-1002. 


\title{
RESUMO
}

Intensificação Negociada: Mudanças no Regime de Trabalho de uma Grande Empresa Varejista no Chile (2006-2018).

Apesar da precariedade e o declínio geral da atividade sindical, têm sido redescobertas experiências de revitalização e contramovimentos desde os trabalhadores, os que se apresentam como feitos heroicos e sem efeitos estruturantes sobre as condições de trabalho, em um panorama de mercantilização progressiva da sociedade. A partir do estudo de caso de Walmart no Chile, é sustentado que a atividade sindical ao interior da empresa tem promovido a transformação de seu regime de trabalho, passando de um "despótico" baseado na inseguridade salarial, o abuso e as práticas anti-sindicato, a outro "hegemônico segmentado", com melhores tratos, tentativas de controle normativo, fomento da sindicalização e benefícios materiais desiguais. Conclui-se reafirmando as teses de que a organização autônoma dos trabalhadores é possível em contextos de precarização, que a precariedade é reversível e que o controle do trabalho no retalho tem limites de reprodução sob mecanismos puramente despóticos e de anti-sindicato.

Palavras-chave: regime de trabalho; sindicatos; varejo; compromisso de classes

\begin{abstract}
The Negotiated Intensification: Changes in the Work Regime of a Large Retail Company in Chile (2006-2018)
\end{abstract}

Despite the precariousness and the general decline in union activity, experiences of revitalization and countermovement of workers have been rediscovered, which are presented as heroic deeds and without structuring effects on working conditions, in a scenario of progressive commercialization of society. Based on the case study of Walmart in Chile, it is argued that union activity within the company has promoted the transformation of its work regime, going from a "despotic" one, based on wage insecurity, abuse, and practices anti-union, to another, "segmented hegemonic", with better treatment, attempts at regulatory control, promotion of unionization and unequal material benefits. The article concludes by reaffirming the thesis that the autonomous organization of workers is possible in contexts of precariousness, that precariousness is reversible, and that the control of work in retail has reproduction limits under purely despotic and anti-union mechanisms.

Keywords: Work Regimen; Unions; Retail; Class Commitment. 


\section{RÉSUMÉ \\ L'escalade Négociée: Modifications du Régime de Travail ans une grande Entreprise de Vente au Détail au Chile (2006-2018)}

Malgré la précarité et le déclin général de l'activité syndicale, des expériences de revitalisation et de contre-mouvements ont été redécouvertes par les travailleurs, présentées comme des actes héroïques et sans effets structurants sur les conditions de travail, dans un panorama de commercialisation progressive de la société. Sur la base de l'étude de cas de Walmart, au Chili, il est avancé que l'activité syndicale au sein de l'entreprise a favorisé la transformation de son régime de travail, passant d'un régime «despotique» basé sur l'insécurité salariale, les abus et les pratiques antisyndicales à un autre "d'hégémonie segmentée", avec un meilleur traitement, des tentatives de contrôle réglementaire, la promotion de la syndicalisation et des avantages matériels inégaux. Il conclut en réaffirmant la thèse que l'organisation autonome des travailleurs est possible dans des contextes de précarité, que la précarité est réversible et que le contrôle du travail dans le commerce de détail a des limites de reproduction sous des mécanismes purement despotiques et antisyndicaux.

Mots-clés: Régime de travail; Syndicats; commerce de détail, Engagement de classe

\section{RESUMEN}

La Intensificación Negociada: Cambios en el Régimen de Trabajo de una Gran Empresa del Retail en Chile (2006-2018)

Pese a la precarización y declinación general de la actividad sindical, se han redescubierto experiencias de revitalización y contramovimientos desde los trabajadores, los que se presentan como gestas heroicas y sin efectos estructurantes sobre las condiciones de trabajo, en una panorama de mercantilización progresiva de la sociedad. A partir del estudio de caso de Walmart en Chile, se sostiene que la actividad sindical al interior de la empresa ha promovido la transformación de su régimen de trabajo, pasando de uno "despótico" basado en la inseguridad salarial, el abuso y las prácticas antisindicales, a otro "hegemónico segmentado", con mejores trato, intentos de control normativo, fomento de la sindicalización y beneficios materiales desiguales. Se concluye reafirmando las tesis de que la organización autónoma de los trabajadores es posible en contextos de precarización, que la precariedad es reversible, y que el control del trabajo en el retail tiene límites de reproducción bajo mecanismos puramente despóticos y antisindicales.

Palabras claves: régimen de trabajo; sindicatos; retail; compromiso de clases 